\title{
Design and Structural Analysis of Scissor Jack using ANSYS Workbench
}

\author{
C. Sai Kiran ${ }^{1}$ and J. Sruthi ${ }^{2}$ \\ ${ }^{1}$ Asst. Professor, CVR College of Engineering/Mechanical Engg. Department, Hyderabad, India \\ Email: csaikiran001@gmail.com \\ ${ }^{2}$ Asst. Professor, CVR College of Engineering/ Mechanical Engg. Department, Hyderabad, India \\ Email: sruthij02@gmail.com
}

\begin{abstract}
In this paper, a scissor jack was designed and analyzed which can be used for lifting the vehicle in vertical direction. A scissor jack is an example of a power screw in which a small force is applied in a horizontal plane for raising or lowering a large load. The purpose of this work is to design a scissor jack for safe, easy operation and which will able to lift the car without spending much effort, by studying the total deformation and von-mises stress values of the scissor jack which are useful for assessing the safety and life prediction of the scissor jack. A detailed structural analysis of scissor jack is performed using ANSYS and this analysis helps to predict which parts of the scissor jack fails when a certain external load acts on it.
\end{abstract}

Index Terms: Scissor Jack, Power Screw, Von-mises stress, ANSYS.

\section{INTRODUCTION}

Whenever a vehicle tyre fails while travelling, the driver must lift the vehicle from the ground level and perform repair or maintenance of that vehicle. Therefore, a jack is used in case of a vehicle emergency. A jack is a mechanical device used for lifting heavy loads or to apply great forces. A jack is a device used for raising all or part of a vehicle into the air to facilitate repairs of that vehicle. A mechanical jack has screw threads for lifting heavy equipment or vehicles. A car jack has mechanical advantage which allows lifting a vehicle by manual force. The most common forms of jacks are scissor jack, screw jack, floor jack or garage jack which lifts vehicles for maintenance or repairs. Jacks are usually rated for a maximum lifting capacity. In industries, jacks are rated for carrying tons of load.

Manoj et al. [1] has created a scissor jack model for carrying actual loads of a light motor vehicle. The scissor jack is modelled in Pro-e software and analysed in ANSYS software. Welded joints are replaced with rivets for reduction of weight in the material. The main objective is to reduce the weight of the scissor jack by changing manufacturability and scissor jack should withstand the strength requirements. Loads are applied on the scissor jack and yield stress, failure strain and plastic strain values are obtained using ANSYS software and the values are within the limits.

Chetan et al. [2] has optimized scissor jack by designing and analysing the scissor jack model for different loading conditions. Different failure analysis of a scissor jack is performed to study the performance and reliability of the scissor jack. A scissor jack is tested under different loading conditions and the scissor jack failure is observed in screws, arm teeth, jack head failure etc. Therefore, a new model of screw jack is created with the maximum load carrying capacity and changing the angle between lifting arms of the screw jack.

Dhamak et al. [3] has designed, optimized and standardized a scissor jack to avoid field failure by modelling scissor jack in CATIA software and standardized by using MATLAB software. The design of scissor jack is modified based on human factor considerations and its functionality. The different failure modes of scissor jack are analysed and redesign of scissor jack is made interms of cost and quality of the scissor jack. Inclined load inspection test and stability test on vehicle is performed by using scissor jack.

Jaideep et al. [4] have taken the reference of Mahindra bolero scissor jack. The dimensions of the scissor jack were measured by using Vernier callipers. Different parts of scissor jack are modelled and assembled in CATIA and different parameters like maximum shear stress, maximum principal tensile stress, total torque required to lift the vehicle etc. are calculated for the components of scissor jack to avoid failure. To avoid failure of the scissor jack, different stresses are analysed and all the results were verified and they are within the safe limits.

Xin et al. [5] has installed damping device for the scissor jack system for good compactness. The design process and theoretical basis comparison is used for finding optimum geometry parameter. The scissor damping system uses minimum number of dampers to obtain damping coefficient. The scissor jack damper can provide a significant increase in the damping ratio while reducing both the drift and acceleration response. The relative velocities and displacements of the structure are observed, and the resulting damper force is amplified. The result shows that the force exerted on the structure is larger than the force produced by traditional diagonal braced.

Haribaskar et al. [6] has modified the conventional screw jack to remote controlled scissor jack which helps to lift the load by using motor as a source of energy. The scissor jack must be compact and light weight for storing it in an automobile trunk.

Chiang et al. [7] has summarized results from an evaluation program and tests performed by the National Highway Traffic Safety Administration which involves 120 automobile scissors jack selected from 24 models made by 14 different manufacturers. A mathematical model of different types of scissor jacks is introduced to describe the load and supporting characteristics. The four major failure 
modes of the scissor jack are: unable to raise or lower the vehicle, collapsing, penetration of the vehicle frame and toppling over. The analytical result values are same as the result of the tests performed.

Jaideep et al. [8] has modelled a scissor jack for changing tyres or for maintenance purpose of a car. Scissor jack will convert the rotary motion into reciprocating motion. They have calculated the design criteria of different parts of a scissor jack analytically and the results obtained from CAE tools like ANSYS have been compared by taking three different materials for two different sections to find out which material has less deformation and to sustain high yield strength without any failure. The objective is to reduce the weight with high strength of scissor jack and to calculate the stresses like maximum principal stress, maximum shear stress, bending stress, torsion stress and crushing stresses which are induced in the scissor jack by comparing it with the analysis performed using ANSYS software. They found that the values of ASTM A36 mild steel are more equivalent in designing and modelling the scissor jack based on its minimum deformation and high load sustainable capacity without causing any type of failure in the scissor jack.

Nitinchandra et al. [9] has modelled a toggle jack which is used to lift heavy loads. The main parts are nut and screw. The nut is a stationary part and the screw is a moving part. The bearing stress induced in the materials causes the failure in the nut and stresses like shear and tensile cause the failure of the screw in the scissor jack. The objective is to select a pair of material for screw and nut which can resist the induced stresses by application of different loading conditions varying from $1 \mathrm{KN}$ to $5 \mathrm{KN}$. The design of screw, nut and links of the scissor jack are performed by taking different combination of materials. Comparison of design stresses and calculated stresses acting on the nut and screw is performed. The results are within the limits.

Uday et al. [10] has designed a pneumatic scissor jack which can lift a car when it is placed at the bottom of the vehicle. The main parts are toggle jack with power screw, gear pair and DC wiper motor. The designed scissor jack can lift 4,000-5,000 pounds vehicle from the ground.

\section{A. Different Types of Jacks}

Jacks are used for lifting heavy weights by applying a force on it. The different names for the jacks are given depending upon the design, utility, technology used and customization etc. Some of the important jacks used for lifting are given below:
1. Scissor jack.
2. Hydraulic jack.
3. House jack/Screw jack.
4. Pallet jack.
5. Floor jack.
6. Pneumatic jack.
7. Bottle jack.
8. Farm jack.

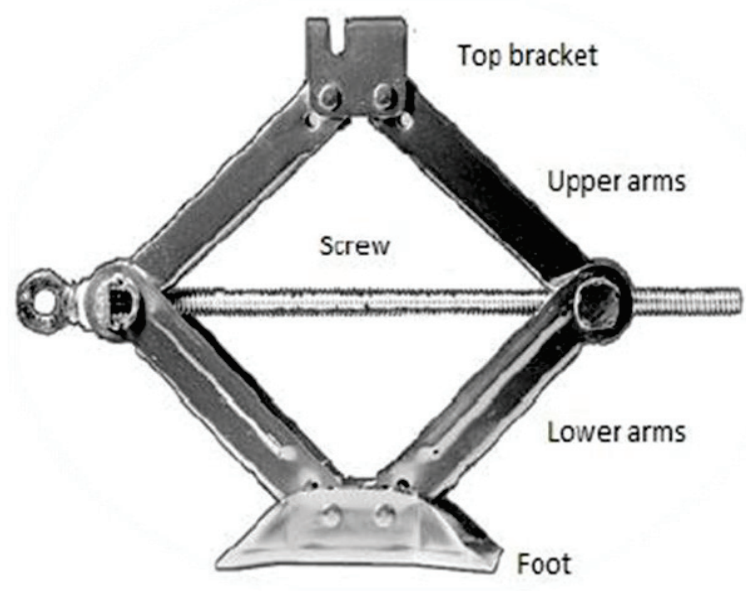

Figure 1. Scissor Jack

Scissor jack is represented in Fig. 1. The main parts of a scissor jack are top bracket, foot, screw, upper and lower arms.

\section{B. Construction of a Scissor Jack}

All the different scissor jacks are similar in design which consists of a base plate, four lifting arms, a carrier plate, eight connection pins, two connection members, a crank and a power screw. The four metal pieces are connected to each other at the corners with a bolt. A scissor jack is having a diamond shape frame with a nut at one side and a sleeve on the other side. The nut and sleeve are supported by a screw. When the screw is rotated, the nut moves away or towards from the sleeve depending on the direction of the rotation. The rotation will either lift-up or lower down the vehicle which is supported on the scissor jack. All the different parts of the scissor jack are shown in Fig.2.

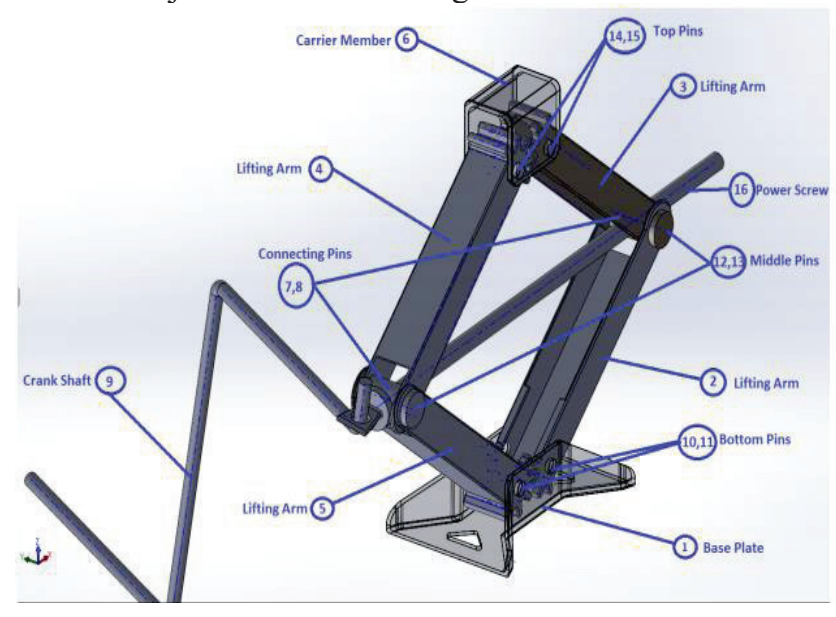

Figure 2. Scissor jack and its parts

\section{Scissor Jack Material}

Scissor jacks are usually made of materials that are very strong and are suitable for withstanding heavier loads on it. Steel is selected as material for scissor jack because of its durability. 
TABLE I.

MATERIAL Properties OF STEEL

\begin{tabular}{|c|c|c|}
\hline S. No & Properties & Steel \\
\hline 1. & Density $\left(\mathrm{Kg} / \mathrm{m}^{3}\right)$ & 7,800 \\
\hline 2. & Young's Modulus (Mpa) & 210000 \\
\hline 3. & Yield Strength (Mpa) & 360 \\
\hline 4. & Tensile Strength (Mpa) & 610 \\
\hline 5. & Poisson's Ratio & 0.3 \\
\hline
\end{tabular}

\section{Modelling ANd Finite Element ANAlysiS}

\section{A. Scissor Jack Model}

All the different parts of the scissor jack are modelled in SOLIDWORKS software by using different commands and all the individual parts of the scissor jack are assembled in the SOLIDWORKS as shown in Fig. 3

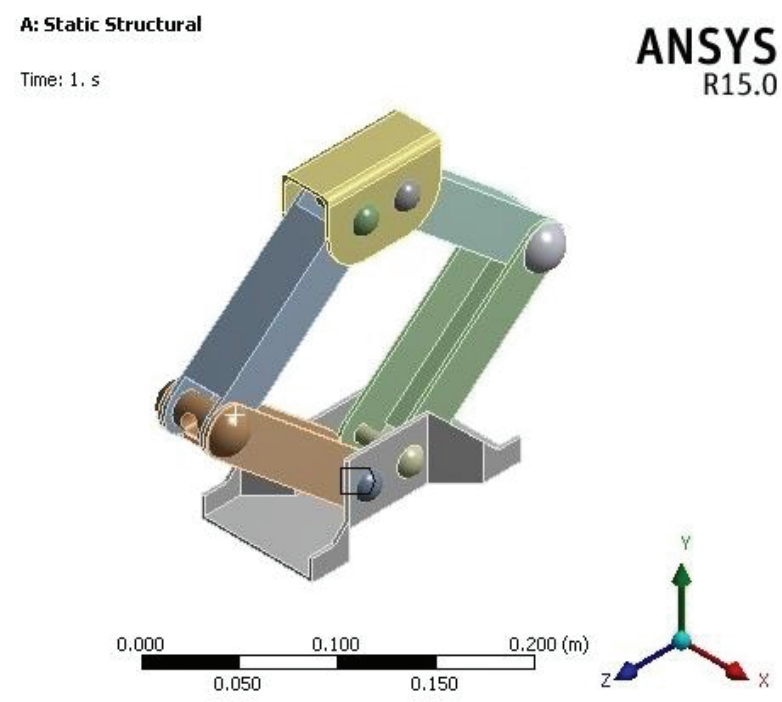

Figure 3. Assembled Model of Scissor Jack

\section{B. Elememt Type}

The element type used is SOLID 186. SOLID 186 is a higher order 3-D with 20-node having three degrees of freedom per node, solid element that exhibits quadratic displacement behavior. This element supports large deflections, plasticity, large strain capabilities, hyper elasticity, stress stiffening and creep.

\section{Meshing}

After assigning element type to the scissor jack, the created solid model is converted into IGES format and imported into ANSYS Workbench. Meshing is an important process of an analysis and it should be performed on the scissor jack model. Meshing is the process of dividing the created model in number of divisions or elements which consists of nodes. By meshing process, we can determine the efficiency and effectiveness of any analysis. An automated mesh generation is as shown in Fig. 4.

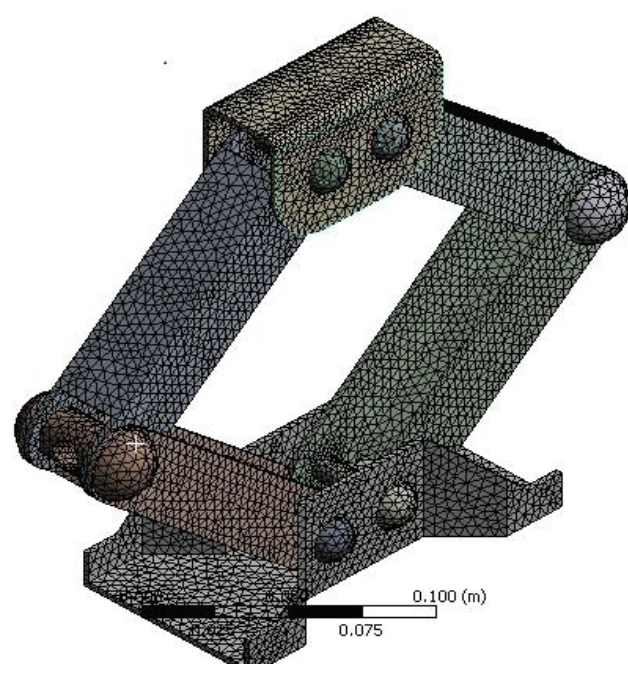

ANSYS

R15.0

Figure 4. Meshing of Scissor Jack

Meshing is applied by using automatic mesh. Under mesh sizing, mesh was set to fine mesh to achieve accurate and precise results. Rather than using a fine mesh all over the components, coarse mesh was used on larger area and fine mesh was used at the area of stress concentration.

\section{Applying Loads}

In the analysis setting, fixed support is assigned to the base plate of the scissor jack, by assigning the fixed support the base plate is constrained in all degrees of freedom and it would withstand the forces acting on the scissor jack as shown in Fig. 5.

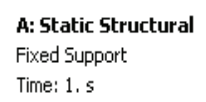

\section{Fixed Support}
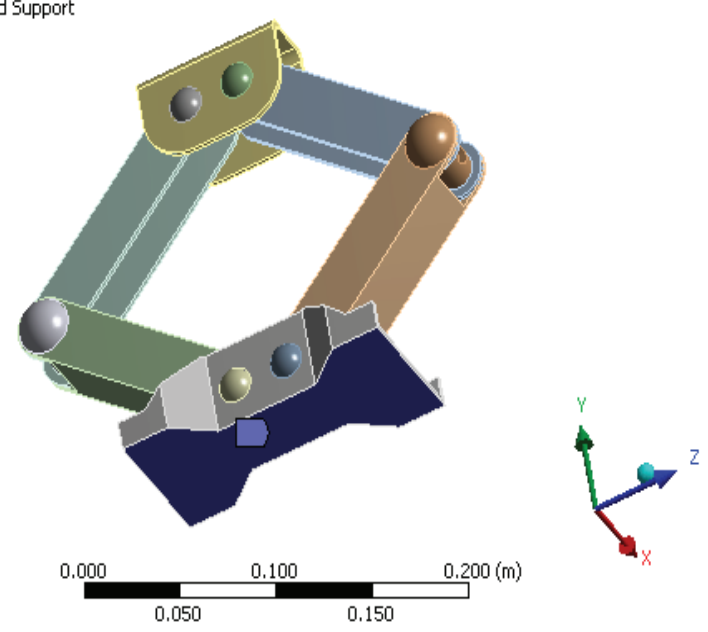

Figure 5. Fixing the base of Scissor Jack

The average weight of the car is calculated and converted into force. A force of $2943 \mathrm{~N}$ is applied on the top bracket of the scissor jack as shown in Fig. 6. 


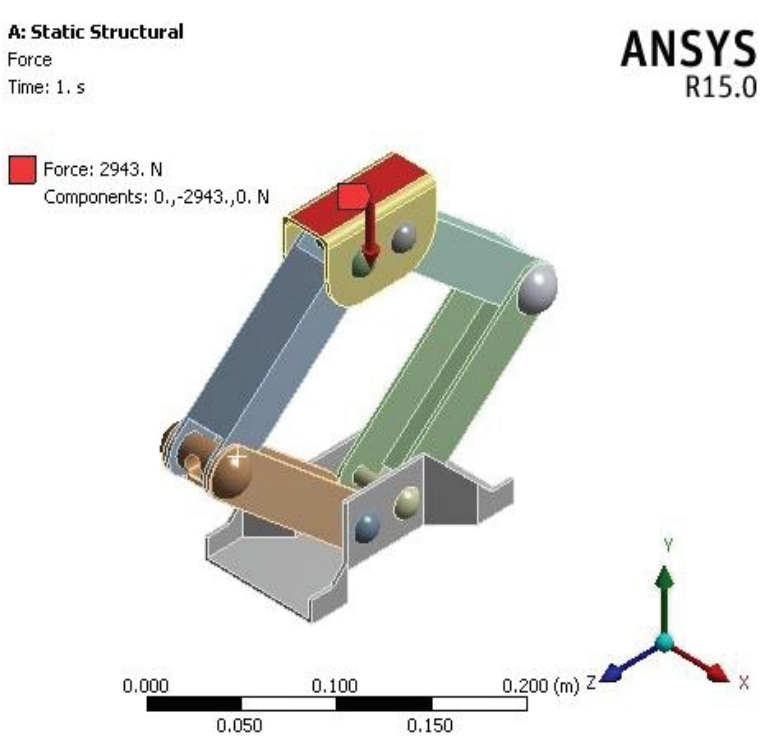

Figure 6. Applying Force on Top Bracket

\section{RESULTS AND Discussions}

After fixing the base and applying the load on the top bracket of the scissor jack, structural analysis is performed on the scissor jack in ANSYS workbench. The following results were observed in the structural analysis.

\section{A. Total Deformation of Scissor Jack}

After performing structural analysis on the scissor jack by applying the load on it, maximum total deformation of 0.115 $\mathrm{mm}$ is observed from the Fig. 7.

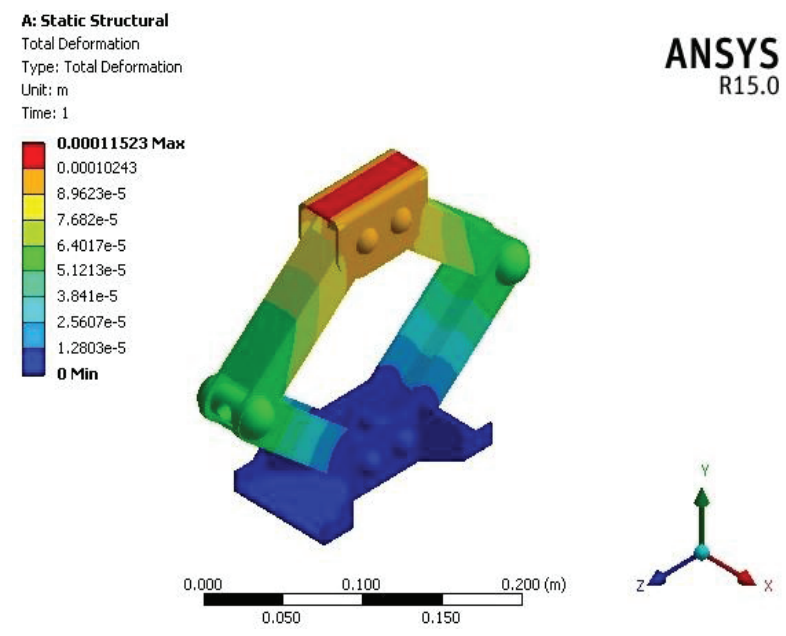

Figure 7. Total Deformation of Scissor Jack

\section{B. Von-mises Stress of Scissor Jack}

After performing structural analysis on the scissor jack by applying the load on it, maximum von-mises stress of 128.21 Mpa and minimum von-mises stress of $6.868 \mathrm{Mpa}$ is observed in the scissor jack from the Fig. 8.

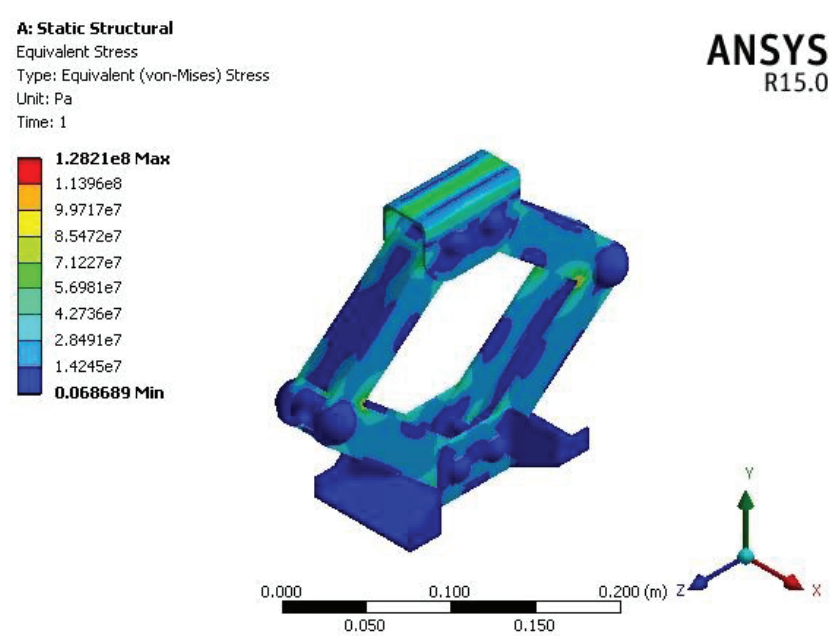

Figure 8. Von-mises Stress of Scissor Jack

\section{CONCLUSIONS}

In this paper, a scissor jack is modelled in SOLIDWORKS and a structural analysis of scissor jack with a force of $2943 \mathrm{~N}$ is performed in ANSYS software. The total deformation and von-mises stress of the scissor jack is analysed. From the results, it is observed that the maximum deformation and the von-mises stress of the scissor jack are within the limits. Therefore, modelled scissor jack is safe to use and has long life.

In future scope, different types of jacks can be modelled by taking different materials and by giving different loading conditions for structural analysis.

\section{REFERENCES}

[1] Manoj R Patil and S D Kachave, "Design and analysis of scissor jack," International Journal of Mechanical Engineering and Robotics Research, vol. 4, pp. 327-335, January 2015.

[2] Chetan S. Dhamak, D.S. Bajaj and V.S. Aher, "Design and optimization of scissor jack," International Journal of Advances in Production and Mechanical Engineering, vol. 2, pp. 29-34, 2016.

[3] C.S. Dhamak, D.S. Bajaj, V.S. Aher and G. Nikam, "Design and standardization of scissor jack to avoid field failure," International Journal of Advance Research and Innovative Ideas in Education, vol. 1, pp. 1-10, 2015.

[4] Jaideep Chitransh and Dilshad Hussain, "Designing and calculating the stresses induced in scissors jack for three different materials," International Journal of Scientific and Technology Research, vol. 5, pp. 119-123, July 2016.

[5] Xin Zhao and Zhuang MA, "Optimization of scissor-jackdamper's parameters and performance under the constrain of human comfort," Advances in Civil, Environmental, and Materials Research, August 2016.

[6] Haribaskar, G. Dhenesh Kumar, Arun Kumar.C, Hari Haran.P, Boobalan.M and Sadha Sivam.C, "Remote controlled scissor jack to lift the vehicle," International Journal of Advance Research, vol. 3, pp. 1279-1282, 2015.

[7] G. D. C. Chiang, "Test, evaluation and failure analysis of automotive scissors jack," Journal of Vibration, Acoustics, Stress and Reliability in Design, vol. 109, pp. 207-213, November 2009. 
[8] Jaideep Chitransh and Akash Singh Patel, "Comparing mathematical model of scissors jack analytically and by using CAE tools," International Journal of Computational Engineering Research, vol. 6, pp. 49-53, November 2016.

[9] Nitinchandra R. Patel, Sankethkumar Dalwadi, Vijay Thakor and Manish Bamaniya, "Design and toggle jack considering material selection of screw - nut combination," International Journal of Innovative Research in Science and Technology, vol. 2, pp. 1748-1756, May 2013.

[10] Pandra Uday Kumar, and G. Ramanjulu, "Design and analysis of center jack for cars," International Journal \& Magazine of Engineering, Technology, Management and Research, vol. 2, pp. 1476-1479, September 2015. 